\title{
Towards an optimized design for the Cherenkov Telescope Array
}

\author{
V. Stamatescu*, Y. Becherini ${ }^{\dagger}, * *$, K. Bernlöhr ${ }^{\ddagger}$, E. Carmona ${ }^{\S}$, P. Colin ${ }^{\mathbb{I}}$, C. \\ Farnier", L. Gerard ${ }^{\dagger \dagger}$, J. A. Hinton ${ }^{\dagger}$, B. Khélifi**, N. Komin ${ }^{\S}$, G. \\ Lamanna $^{\S \S}$, J.-P. Lenain ${ }^{\text {IUII }}$, G. Maier ${ }^{\dagger \dagger}$, A. Moralejo*, C. L. Naumann ${ }^{* * *}$, R. \\ D. Parsons ${ }^{\ddagger}$, F. Di Pierro ${ }^{\dagger \dagger}$, H. Prokoph ${ }^{\dagger \dagger}$ and S. Vorobiov ${ }^{\dagger \dagger}$ \\ for the CTA Consortium \\ ${ }^{*}$ IFAE Barcelona, ${ }^{\dagger}$ APC Paris, ${ }^{* *}$ LLR Paris, ${ }^{\ddagger}$ MPI-K Heidelberg, ${ }^{\S}$ CIEMAT Madrid, \\ ${ }^{\mathbb{I} M P I-P}$ Munich, "Stockholm University, ${ }^{\dagger} D E S Y$ Zeuthen, ${ }^{*}$ Univ. of Leicester, ${ }^{\S}$ LAPP Annecy,

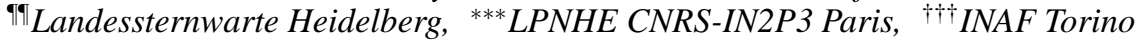

\begin{abstract}
The Cherenkov Telescope Array (CTA) is a future instrument for very-high-energy (VHE) gamma-ray astronomy that is expected to deliver an order of magnitude improvement in sensitivity over existing instruments. In order to meet the physics goals of CTA in a cost-effective way, Monte Carlo simulations of the telescope array are used in its design. Specifically, we simulate large arrays comprising numerous large-size, medium-size and small-size telescopes whose configuration parameters are chosen based on current technical design studies and understanding of the costs involved. Subset candidate arrays with various layout configurations are then selected and evaluated in terms of key performance parameters, such as the sensitivity. This is carried out using a number of data analysis methods, some of which were developed within the field and extended to CTA, while others were developed specifically for this purpose. We outline some key results from recent studies that illustrate our approach to the optimization of the CTA design.
\end{abstract}

Keywords: gamma-rays: observations, instrumentation: detectors

PACS: 95.45.+i, 95.55.-n, 95.55.Ka, 95.75.-z, 95.85.Pw

\section{PERFORMANCE OF THE CANDIDATE ARRAYS}

We evaluate the performance of CTA candidate arrays, which are compared in Fig. 1 in terms of their differential sensitivity, calculated using our baseline analysis [1]. The candidate array layouts comprise varying numbers of large-size (LSTs), mid-size (MSTs) and small-size (SSTs) telescopes that are selected according to a fixed estimated total cost from a large 275-telescope configuration known as production 1 (prod-1). Part of the simulations were performed by using a distributed approach within the European Grid Initiative [2]. For the upcoming prod-2, a Grid-based simulation and analysis pipeline is being prepared. Arrays E and I are so-called balanced arrays (balanced in terms of the distribution resources across energy range of CTA), and thus are well-suited for evaluating the effects of different analysis methods or telescope designs.

We verify that configurations selected to be optimal using the baseline analysis also reach close to the optimal sensitivity when employing alternative analysis methods (see descriptions in [1,3]). Fig. 1 illustrates the effect of these methods on sensitivity for the array I, which consists of 3 LSTs each with $4.9^{\circ}$ field of view (FoV), 18 MSTs with $8^{\circ} \mathrm{FoV}$, and 56 SSTs with $9^{\circ}$ FoV. While most methods are under development, some 

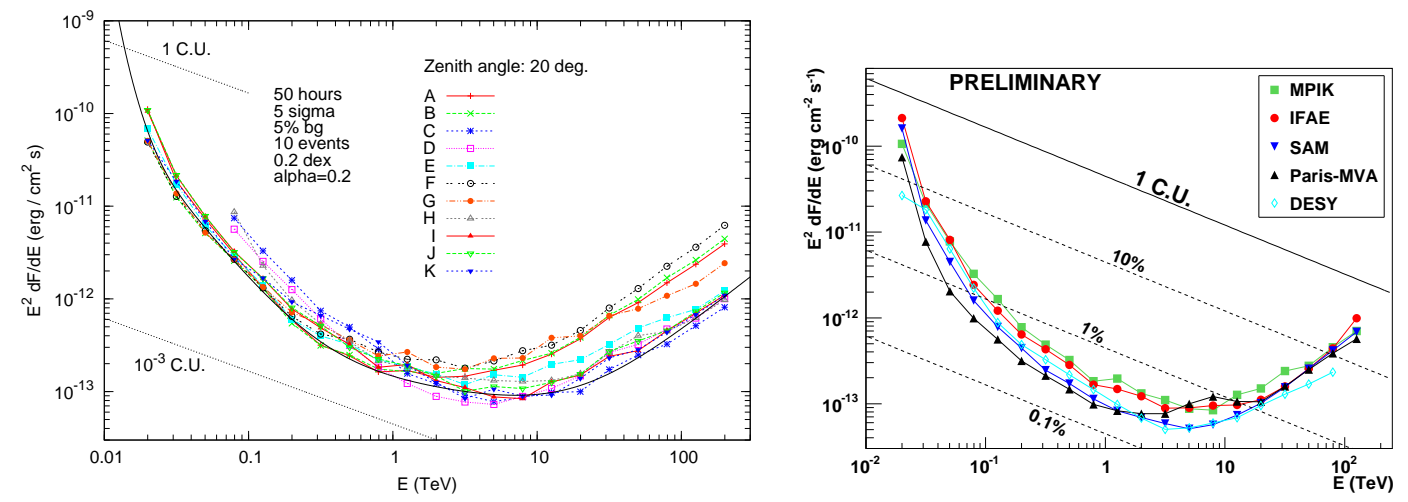

FIGURE 1. (Left) Point-like source sensitivity of $11 \mathrm{CTA}$ candidate array layouts of identical estimated costs for 50 hours observation time and zenith angle of $20^{\circ}$, evaluated with the baseline analysis method. The solid black line approximates the best performance of any of these arrays at any energy, excluding D, which is optimized for energies of a few TeV. The Crab Unit (C.U.) and milli-C.U. fluxes (see text) are shown for comparison. (Right) Sensitivity of balanced array I, evaluated using the baseline/MPIK (green squares) IFAE (red circles), SAM (blue triangles), Paris-MVA (black triangles) and DESY (light-blue diamonds) analyses.

already approach differential sensitivity levels of 2 milli-C.U. ${ }^{1}$ (Crab Unit) in the core range around $1 \mathrm{TeV}$. This minimum detectable flux is determined conservatively, using 5 bins per decade in energy and demanding, for a given bin, a minimum $5 \sigma$ detection (using Eq. 17 from Li \& Ma [4] and 5 background control regions), $\geq 10 \gamma$-ray events, and that the $\gamma$-ray excess be greater than $5 \%$ of the residual background.

We are also investigating the off-axis performance of CTA. Fig. 2 shows the off-axis differential sensitivity for offset angles out to $3^{\circ}$ calculated using two analyses for array E, which comprises 4 LSTs with $4.6^{\circ}$ FoV, 23 MSTs with $8^{\circ} \mathrm{FoV}$, and 32 SSTs with $10^{\circ}$ FoV. While the off-axis analysis algorithms are expected to have room for improvement, these results indicate that the point-source differential sensitivity in the energy range of $\sim 100 \mathrm{GeV}$ to $\sim 10 \mathrm{TeV}$ may be well below $0.1 \mathrm{C}$.U. out to offsets of at least $\sim 3^{\circ}$ off-axis.

\section{LST OPTICS AND CAMERA DESIGN}

The LSTs are simulated in prod -1 with a parabolic dish shape of focal length $f=31.2 \mathrm{~m}$ and area $A=412 \mathrm{~m}^{2}$, coupled with $0.09^{\circ}$ pixels. Mechanical constraints of the envisaged LST structure impose a maximum $f=28 \mathrm{~m}$. In a dedicated study, we simulate an alternative design with $A=382 \mathrm{~m}^{2}$ and $0.1^{\circ}$ pixels, and use an intermediate dish shape to mitigate the degradation of the optical PSF given by the reduced $f / D$, where $D$ is the mirror diameter. We also simulate an idealized design with $A=382 \mathrm{~m}^{2}, f=37 \mathrm{~m}$ and $0.06^{\circ}$ pixels. Results show that the mean Hillas width [5] of small gamma-ray images, with Hillas sizes of around 100 p.e., are $20 \%$ smaller for the idealized case. However, this

11 C.U. $=2.79 \cdot 10^{-7} \mathrm{~m}^{-1} \mathrm{~s}^{-1} \mathrm{TeV}^{-1} \times(E / T e V)^{-2.57}$ 

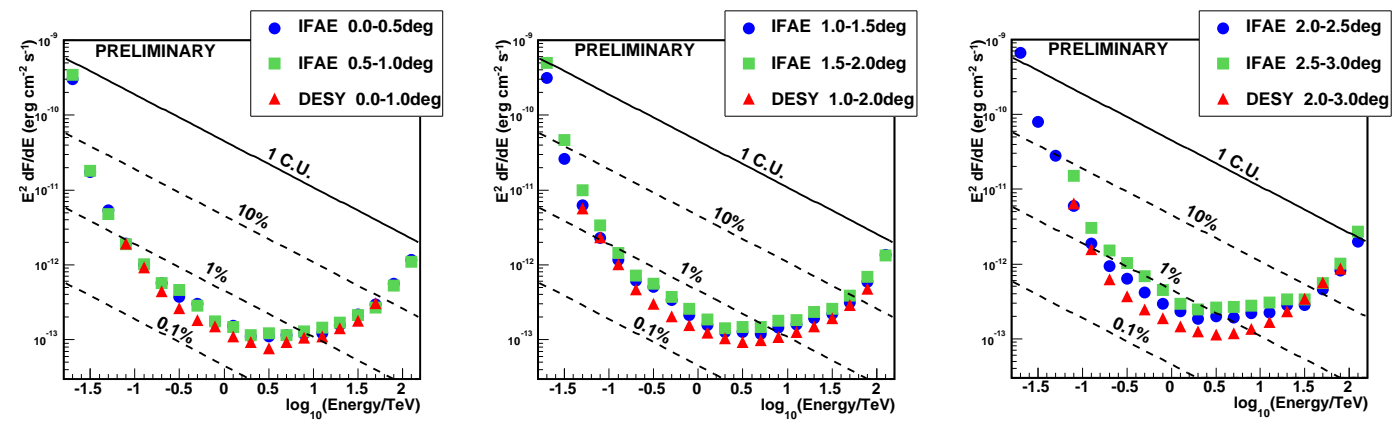

FIGURE 2. Offset point-like source 50 hour sensitivity of balanced array E, for source offsets of between $0-1^{\circ}$ (left), $1-2^{\circ}$ (middle) and $2-3^{\circ}$ (right), evaluated using two alternative analysis methods: IFAE (blue circles and green squares) and DESY (red triangles).
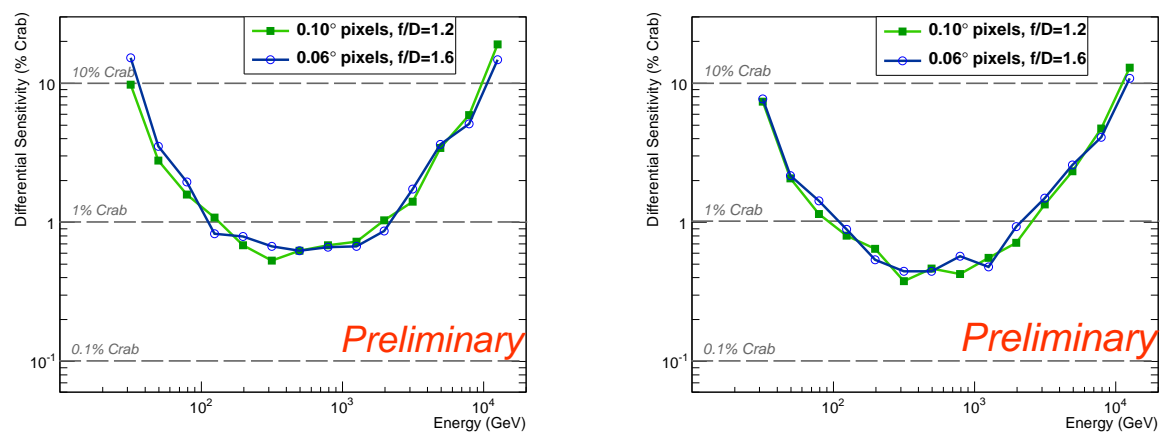

FIGURE 3. Differential sensitivity of (left) 3 LSTs arranged in an equilateral triangle and (right) 4 LSTs in a square, both of side length of $105 \mathrm{~m}$, for realistic (green squares) and ideal (blue open circles) LST designs (see text) of the camera pixel size and the telescope optics. Note that the contribution to the background from electrons has not been taken into account in this LST study.

does not translate to an improvement in gamma-hadron separation power, as indicated in Fig. 3, which compares the realistic and idealized designs of two LST arrays.

The LST $f / D$ and FoV parameters are being tuned for prod-2 by using a toy model, which estimates the relative sensitivity in the LST-dominated energy range of $30-100$ $\mathrm{GeV}$, at a fixed LST array cost. The model is based on prod-1 simulations and assumes that the background control region size depends on the angular distance between the source position and camera center, and that sensitivity scales as $N^{-0.5}$, where $N$ is the number of LSTs. Based on the relative sensitivity tradeoff between $f / D$ and FoVs, we find, for point-like sources, that optimal values are close to $f / D=1.2$ and $\mathrm{FoV}=4.2^{\circ}$. For extended sources $\left(\sim 1^{\circ}\right)$, the optimal values become larger: $f / D=1.4$, FoV $=4.5^{\circ}$.

\section{OBSERVATIONS UNDER PARTIAL MOONLIGHT}

Observations under moonlight conditions permit to extend the observing time by as much as 30\%, based on experience with MAGIC [6] and VERITAS, and are important 
for the detection of transient phenomena. To estimate the performance of CTA under moonlight conditions, MC simulations with a factor 4.5 higher night sky background (NSB) have been performed. This corresponds to nights with the Moon above the horizon, illuminated at $\sim 60 \%$. In the core energy range around $1 \mathrm{TeV}$, analysis results indicate a similar performance to the one for dark sky conditions, in terms of sensitivity, angular resolution and energy resolution. However this is not the case for the low energy regime. Here, due to the higher noise levels, the energy thresholds are generally a factor of 2 higher, although this number depends on the array layout.

\section{READOUT SIMULATIONS}

A number of tools exist to simulate the optical and electronic designs being evaluated within CTA (see [1] for a brief overview). Here we outline ongoing studies that focus on the design of the camera readout. One such study [7] aims to optimize the pixel integration time window, which, given the short time scale of Cherenkov light flashes, can minimize the error in collected pixel signal charge over NSB fluctuations. For short integration times, the pixel charge resolution degrades due to Cherenkov light fluctuations, while for longer times the NSB dominates. A dynamic integration window, whose duration varies as a function of signal amplitude, may improve charge resolution over a fixed duration window. Under dark sky conditions, any improvements are expected to be very small, as the Poissonian fluctuations of the Cherenkov signal dominate. However under partial moonlight or Galactic plane observations more significant improvements can be envisaged. A complementary study [8] proposes a flexible readout scheme in which only the pixels around the core of the shower image are recorded, with the aim of reduced data rates and effective dead times. The scheme would employ lower threshold local trigger information to define regions of interest in the camera and follow the shower temporal development, while a higher threshold trigger would trigger the camera readout.

\section{ACKNOWLEDGMENTS}

We gratefully acknowledge support from the agencies and organizations listed in this page: http: / / www. cta-observatory .org/ ?q=node/22.

\section{REFERENCES}

1. K. Bernlöhr et al, upcoming Special Issue on CTA of Astropart. Phys. (2012).

2. N. Komin et al., http://hal . archives-ouvertes.fr/hal-00653017 (2011)

3. Y. Becherini, B. Khélifi, S. Pita, M. Punch et al., Advanced analysis and event reconstruction for the CTA Observatory, these proceedings (2012).

4. T.P. Li \& Y.Q. Ma, ApJ 272317 (1983).

5. A.M. Hillas, Proc. $19^{\text {th }}$ ICRC Vol. 3, 445 (1985)

6. D. Britzger et al., Proc. $31^{\text {st }}$ ICRC 2009 (arXiv:0907.0973)

7. S. Vorobiov, et al., NIM A, DOI : 10.1016/j.nima.2011.10.048 (2011).

8. C. L. Naumann et al, NIM A, DOI : 10.1016/j.nima.2011.11.008 (2011). 